\title{
Desnutrición e infancia en Argentina: Dimensiones, tendencias y miradas actuales sobre el problema a partir de la combinación de un diseño observacional y cualitativo
}

\author{
Fernando Longhi ${ }^{a, *}$, Alicia Gomez ${ }^{\mathrm{b}}$, Maria Fernanda Olmos ${ }^{\mathrm{b}}$

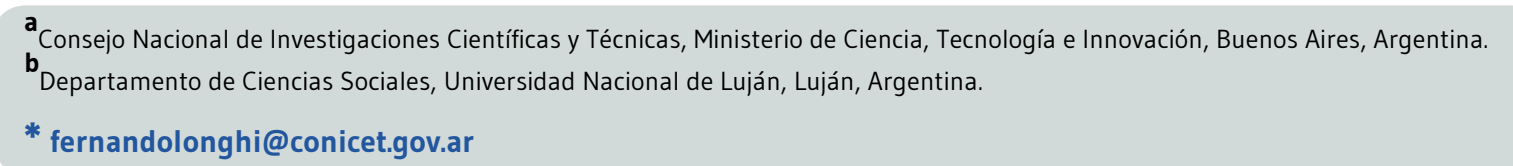

Editora Asignada: Eva María Navarrete Muñoz. Universidad Miguel Hernández. Elche, España.

Recibido el 27 de agosto de 2019; aceptado el 21 de abril de 2020; publicado el 4 de mayo de 2020.

\section{PALABRAS CLAVE}

Trastornos de la Nutrición del Niño;

Desnutrición;

Pobreza;

Argentina.
Desnutrición e infancia en Argentina: Dimensiones, tendencias y miradas actuales sobre el problema a partir de la combinación de un diseño observacional y cualitativo

\section{RESUMEN}

Introducción: La desnutrición infantil constituye la expresión más extrema de la pobreza. Poco se conoce sobre las características de este problema en el marco de un país emergente como lo es Argentina. Este artículo procuró indagar sobre las magnitudes, tendencias, escalas y otras miradas sobre la desnutrición en la niñez en las primeras décadas del siglo XXI, a partir del uso de fuentes no convencionales.

Material y Métodos: Sobre la base de estadísticas de mortalidad, morbilidad y de peso al nacer se elaboraron indicadores de situación nutricional en la niñez. Los mismos fueron consolidados considerando la escala nacional y regional. Se aplicó un abordaje tanto cuantitativo (observacional de orden transversal) como cualitativo (a partir de entrevistas a informantes clave y análisis documental). Se puso énfasis en el uso de fuentes de índole cuantitativo poco utilizadas para la medición de este fenómeno, complementados con otras de orden cualitativo, realizando una crítica valorativa que permite detectar fortalezas y limitaciones de ambos abordajes.

Resultados: Los resultados advierten -en un marco general de descenso- comportamientos diferenciados según regiones y provincias, identificando sectores de magnitudes extremas y tendencias preocupantes en esta materia. Se destaca tanto la persistencia del problema en vastos sectores del Norte argentino, con valores de desnutrición más similares al contexto latinoamericano que al resto del país, así como la emergencia de nuevas problemáticas asociadas.

Conclusiones: Los problemas nutricionales en la niñez argentina se encuentran actualmente atravesados por resabios del pasado -entre ellos la desnutrición-y la emergencia de nuevos problemas, entre los que se destacan el sobrepeso y la obesidad. Los resultados hallados constituyen un capital relevante de insumos para promover acciones concretas en materia de cuidado y protección infantil, diferenciadas según la magnitud, tendencias y localización espacial de determinados problemas nutricionales. En efecto, las provincias del Norte deberían constituir una prioridad para las políticas públicas que busquen atenuar o erradicar los actuales niveles de desnutrición en la niñez. 


\section{KEYWORDS}

Child Nutrition

Disorders;

Malnutrition;

Poverty;

Argentina.
Child malnutrition and childhood in Argentina: Current dimensions, trends and views on the problem from the combination of an observational and qualitative design

\section{ABSTRACT}

Introduction: Child malnutrition is the most extreme expression of poverty. Little is known about the characteristics of this problem in the context of an emerging country such as Argentina. This article sought to investigate the magnitudes, trends, scales and other perspectives on childhood malnutrition in the first decades of the $21^{\text {st }}$ century, based on the use of unconventional sources.

Material and Methods: Based on statistics of mortality, morbidity and birth weight, indicators of nutritional status in childhood were developed. They were consolidated considering the national and regional scale. A quantitative (observational cross-sectional) and qualitative approach was applied (from interviews with key informants and documentary analysis). Emphasis was placed on the use of sources of a quantitative nature little used for the measurement of this phenomenon, complemented by others of a qualitative nature, carrying out a value criticism that allows us to detect strengths and limitations of both approaches.

Results: The results warn -in a general framework of decline- different behaviors according to regions and provinces, identifying sectors of extreme magnitude and worrying trends in this matter. The persistence of the problem in vast sectors of northern Argentina is highlighted, with malnutrition values more similar to the Latin American context than to the rest of the country, as well as the emergence of new associated problems.

Conclusions: Nutritional problems in Argentine childhood are currently crossed by remnants of the past -malnutrition among them- and the emergence of new problems, among which overweight and obesity stand out. The results found constitute a relevant input capital to promote concrete actions in child care and protection, differentiated according to the magnitude, trends and spatial location of certain nutritional problems. In effect, the Northern provinces should be a priority for public policies that seek to mitigate or eradicate the current levels of malnutrition in children.

\section{CITA}

Longhi F, Gomez A, Olmos MF. Desnutrición e infancia en Argentina: Dimensiones, tendencias y miradas actuales sobre el problema a partir de la combinación de un diseño observacional y cualitativo. Rev Esp Nutr Hum Diet. 2020; 24(3): 203-17. doi: 10.14306/renhyd.24.3.933

\section{INTRODUCCIÓN}

Alrededor del año 2010 fue posible observar que, tanto en los países de América Latina en general como en Argentina en particular, se habían registrado grandes avances en el mejoramiento de las condiciones de vida de su población, especialmente si se toma como referencia para la comparación el decenio de los años '90. Dicho proceso reconoce avances de condiciones políticas y económicas que favorecieron el reposicionamiento del Estado en la programación e implementación de políticas públicas ${ }^{1}$. Sin embargo, en los últimos tres años de la primera década de este nuevo siglo se ha registrado cierto estancamiento y/o retrocesos en algunas de las dimensiones fundamentales del bienestar social, especialmente en el caso argentino, entre las que se encuentra la situación nutricional de los niños².
La nutrición -como proceso y fenómeno complejo- está sometida a distintos factores condicionantes; algunos de ellos fijos (como el potencial genético del individuo) y otros dinámicos (como los factores sociales, económicos y culturales, que pueden actuar en forma favorable o desfavorable). Cuando se modifica el equilibrio de estos factores y se ve alterada la nutrición, se interrumpe el crecimiento y desarrollo de los niños, dando lugar a la desnutrición infan$\mathrm{til}^{3}$. En este contexto, como hemos mencionado en estudios anteriores, es conocido que una adecuada nutrición es fundamental para el crecimiento y desarrollo del niño, la deficiencia en su implementación, sobre todo en los primeros años de vida, tiene graves consecuencias para el individuo y la sociedad a la que pertenece $e^{4}$.

Paralelamente a este problema, la Organización Mundial de la Salud (OMS) ha catalogado recientemente a la obesidad 
infantil como la mayor crisis de la salud pública en el mundo. En su informe de 2016 indicaba que se registraron cuarenta y dos millones de niños menores de 5 años con sobrepeso; de ellos, treinta y cinco millones viven en países en desarrollo 5 .

Si bien ambos problemas nutricionales persisten como característicos de las sociedades en desarrollo, las investigaciones académicas de los últimos años han avanzado sobre el estudio del sobrepeso y la obesidad, en detrimento de las problemáticas nutricionales por déficit, las cuales presentan aun una relevancia importante en relación al crecimiento y desarrollo saludable en la niñez. Según estadísticas del Programa Mundial de Alimentos de Naciones Unidas, en América Latina la desnutrición afecta a casi 9 millones de niños menores de 5 años $(16 \%)^{6}$. Se agrega a esta cifra 9 millones más de niños con alto riesgo de desnutrirse debido a las condiciones de pobreza en las que viven.

Los promedios de desnutrición en Argentina son relativamente bajos si se considera el contexto latinoamericano. Sin embargo, ya a inicios del siglo XXI, la FAO destacaba "que, si bien en Argentina la disponibilidad de alimentos es suficiente y aun excedente para cubrir las necesidades energéticas por persona y por día, parte de la población tiene un acceso insuficiente a los alimentos"7. Por otro lado, también UNICEF alertaba a mediados de los noventa que el censo de talla en escolares realizado entre 1991 y 1994 presenta un nivel de retardo de crecimiento elevado en las provincias de Salta, Jujuy, Formosa, Chaco y Misiones (todas en el ámbito del Norte argentino), cuyo panorama se asimilaba más al resto de América Latina que al propio promedio nacional ${ }^{8}$. Otros estudios ${ }^{9,10}$ respaldaban esta evidencia al mencionar que la forma de desnutrición prevalente en Argentina, antes de los 2000, era el déficit de talla o desnutrición crónica, siendo el Norte la región más afectada.

Bajo este contexto de persistencia de diferentes problemas nutricionales en Argentina, este artículo -que opera bajo los lineamientos de un estudio observacional transversalprofundizó en el análisis de la desnutrición infantil y buscó detectar tanto la magnitud de la desnutrición en la niñez como sus principales tendencias, considerando diferentes escalas geográficas y aplicando un abordaje cuantitativo y cualitativo, el primero de ellos amparado en un estudio observacional transverso, según los lineamientos de la guía STROBE (STrengthening the Reporting of OBservational studies in Epidemiology) $)^{11}$.

Se realiza a continuación una breve presentación de los principales métodos, fuentes y resultados para alcanzar el objetivo propuesto, poniendo énfasis en las ventajas y limitaciones de cada uno de los abordajes metodológicos desarrollados y los alcances complementarios del doble abordaje cuantitativo-cualitativo como aporte para el estudio de la morbilidad y la mortalidad por esta causa en el país.

\section{Material y métodos}

Tradicionalmente la desnutrición infantil ha sido evaluada a partir de relevamientos antropométricos, ya sea en el formato de una encuesta nacional de salud o el censo de talla de niños escolarizados, por ejemplo. El uso de indicadores derivados de las Estadísticas Vitales de mortalidad, el Registro de Egresos Hospitalarios o el Registro de Peso en los certificados de nacimiento no ha ocupado un lugar central. A partir de estas fuentes se han generado los indicadores necesarios para responder al objetivo propuesto en la investigación.

En Argentina, estas tres fuentes de datos proporcionan información en forma anual, sistemática y continua. Los nacimientos y las defunciones provienen del registro de estadísticas vitales y la captación de los egresos hospitalarios se realiza a través del registro de servicios de salud. Cada una de estas fuentes proporciona información valiosa que permite abordar la desnutrición en forma cuantitativa, en diferentes niveles de desagregación geográfica. Su análisis ha permitido conocer las manifestaciones del problema de la desnutrición de los menores de 5 años y trazar un perfil de su evolución en diferentes escalas geográficas, así como observar el nivel de asociación que presenta con el contexto sociosanitario.

La tasa de mortalidad por desnutrición se consideró como el indicador más robusto, que proporciona un piso sobre la incidencia del fenómeno. Se usó también el registro de egresos hospitalarios para hacer visible las consecuencias menos trágicas de la desnutrición, a través de considerar el patrón de evolución de la morbilidad por esta causa. El tercer indicador construido derivó de las estadísticas de nacimientos, las cuales proporcionan, entre otras, información sobre el peso al nacer de los niños, el nivel de instrucción de las madres y la duración en semanas de la gestación. De esta manera, los indicadores diseñados fueron:

a) Tasa de mortalidad en la niñez por desnutrición: relaciona las muertes de menores de 5 años cuya causa básica fue la desnutrición en cada año calendario con el total de nacidos vivos de ese año, expresada cada mil nacidos vivos (Periodo 1999-2013). La categoría "Desnutrición y otras anemias nutricionales" está integrada por las siguientes patologías de la CIE 10: 
anemias por falta de hierro (D50), anemia por deficiencia de vitamina B12 (D51), anemia por deficiencia de folatos (D52), otras anemias nutricionales (D53), Kwashiorkor (E40), marasmo nutricional (E41), Kwashiorkor marasmático (E42), desnutrición proteinocalórica (E43-E46), deficiencia de vitamina A (E50), deficiencia de tiamina (E51), pelagra (E52), deficiencia de otras vitaminas del grupo B (E53), deficiencia de ácido ascórbico (E54), deficiencia de vitamina D (E55), otras deficiencias de vitaminas (E56), deficiencia dietética de calcio (E58), deficiencia dietética de selenio (E59), deficiencia dietética de zinc (E60), deficiencia de otros elementos nutricionales (E61), otras deficiencias nutricionales (E63) y secuelas de la desnutrición y de otras deficiencias nutricionales (E64).

b) Tasa de egresos hospitalarios en la niñez por desnutrición: relaciona la cantidad de egresos hospitalarios del sistema público de menores de 5 años según las causas de desnutrición mencionadas con el total de nacidos vivos en cada año calendario, expresada cada mil nacidos vivos (periodo 2000/2005-2011).

c) Una tercera vertiente de aproximación implicó la generación de un indicador que considera el bajo peso al nacimiento (menos de $2.500 \mathrm{~g}$ ) a término proveniente de madres con educación insuficiente a partir de las estadísticas de nacimientos. En este caso se calculó la proporción que representa la cantidad de nacidos vivos provenientes de gestaciones iguales o superiores a las treinta y siete semanas provenientes de madres con nivel educativo igual a primario incompleto, relacionados con el total de nacidos vivos para cada área geográfica.

No obstante, previo a la utilización de la información mencionada, se realizaron exhaustivos análisis de calidad de los registros, tanto en el total del país como para las jurisdicciones provinciales, evaluando las variaciones en la calidad de dichos registros. En forma sucinta, se evaluó la calidad de las estadísticas de:

Mortalidad: Se seleccionaron las variables relevantes para el objeto de estudio del trabajo, a fin de analizar su calidad. Como indicadores de calidad de la información se analizaron, para el período 1999/2013, el porcentaje de casos ignorados de provincia y departamento de residencia, el porcentaje de casos ignorados de edad del fallecido, el porcentaje de casos de causas mal definidas y desconocidas -categoría que agrupa los casos de causa de muerte registrados bajo "signos, síntomas y estados morbosos mal definidos" (R00-R99 de la Clasificación Internacional de Enfermedades 10a Revisión) e ignoradas. Se observó el nivel de error tanto para las defunciones en general como para las defunciones de los menores de un año y los de 0 a 4 años, según su relevancia y pertinencia en el análisis.

Las variables evaluadas fueron: provincia y departamento de residencia, edad del fallecido y causa de muerte (ignoradas y mal definidas).

El lugar de residencia habitual es la localización geográfica o dirección donde reside habitualmente la persona de que se trate. No necesita ser el mismo lugar en que aquella persona se encontraba en el momento en que ocurrió el hecho o su residencia legal. El lugar de residencia se determina, para las defunciones de menores de un año, como el lugar de residencia de la madre en el momento de la muerte del niño (o la del niño si la madre ha muerto). Para el resto de las edades es el lugar de residencia del fallecido al morir.

Los resultados advierten para el total del país que el nivel de omisión de la provincia y el departamento de residencia se mantiene estable en la década, en torno al $2 \%$, lo que evidencia buena calidad de la información. La proporción de casos desconocidos en la edad del fallecido para el total de las defunciones y para los menores de un año, a lo largo de toda la serie de tiempo analizada, y en el total del país, es menor al 0,5\%, indicando también un alto nivel de calidad de la información. Finalmente, el error de la variable "causa de muerte", calculado a partir de la proporción que representan las defunciones registradas bajo "signos, síntomas y estados morbosos mal definidos" muestra, en el total de las defunciones del país, valores relativamente estables, pero en ascenso (entre un $6 \%$ y un $8 \%$ ). El nivel de error de la causa de muerte para las defunciones de los niños menores de 5 años, en total, muestra mejor calidad que en las defunciones totales. Los porcentajes de causas desconocidas o mal definidas en fallecidos de 0 a 4 años de edad son estables alrededor del $6 \%$ durante todos los años del período, aunque se observan diferencias si se analiza el error para las defunciones de los menores de un año y para los de uno a cuatro. Éstos últimos muestran mayores porcentajes de error y bastantes variaciones a lo largo de la serie temporal, aunque no alcanzan los porcentajes más altos observados entre las defunciones totales.

La evaluación de calidad para la edad del fallecido a nivel provincial, según regiones, mostró que es bastante homogénea. Se observan buenos niveles de calidad, consistentes con lo visualizado en el total de defunciones del país. En cambio, la calidad de la información sobre causa de muerte muestra bastante heterogeneidad entre provincias y aunque para el total del país los niveles de casos ignorados o mal definidos rondan entre el 6 y el $8 \%$, los valores son muy superiores en ciertas provincias. Las provincias de Cuyo y la Ciudad de Buenos Aires son las áreas que presentan la 
mejor calidad en la información, con porcentajes de causa de muerte ignorada y mal definida de alrededor del $2 \%$. En el extremo opuesto, la provincia de Santiago del Estero y Tucumán, tienen cerca del $20 \%$ de las defunciones totales registradas como signos, síntomas y estados morbosos mal definidos. Se observa así que la calidad de esta variable es heterogénea por región y provincia y los altos niveles de error, en ciertos casos, denegarían la posibilidad de su utilización.

Egresos hospitalarios: El registro de egresos hospitalarios se encuentra en proceso de mejoramiento, en especial desde el año 2000, pero aún no se observa una consolidación que concentre la información producida, la cual presenta niveles de continuidad, calidad y completitud variables, entre provincias y en el tiempo. En este sentido se presentan dos tipos de falta de información: a) falta total de información para un año por parte de una provincia o b) falta de información de algunos establecimientos de una provincia. Por otra parte, la información disponible en el período de estudio tiene dos momentos: uno puntual referido al año 2000 y otro que va del 2005 a 2011 donde se cuenta con los datos para cada año de este período.

Por ello, los niveles de casos ignorados o no especificados, como indicador de calidad de la información, se analizaron para los años del período 2000-2011, considerando todas las provincias con información parcial y completa, y excluyendo las provincias que no informaron en forma completa en alguno de los años. Se analizó la distribución del error en el total de los egresos hospitalarios y, en los casos pertinentes, para los egresos de los niños de 0 a 4 años, desagregados de 0 años y de 1 a 4 años.

Las variables evaluadas fueron: edad, provincia y departamento de residencia habitual, sexo, días de estadía, diagnóstico principal, tipo de egreso y cobertura en salud (estas dos últimas disponibles a partir del año 2006), nivel de instrucción y situación laboral (estas dos últimas disponibles sólo desde el año 2008, hasta el 2011).

Los resultados permiten detectar que las variables referidas a la provincia y al departamento de residencia habitual presentan una calidad aceptable (niveles de error de menos del $3 \%$ en el caso de la provincia y del $6 \%$ para el departamento de residencia) y también la edad, con alrededor del $2 \%$. Para el diagnóstico principal al egreso, la proporción de error, definida por la cantidad de casos mal clasificados y falta de información en la causa de la hospitalización sobre el total de los egresos, se encuentra en el límite de los parámetros de calidad aceptables, con valores que rondan el $10 \%$. Lo mismo sucede con la variable de cobertura de salud. En el caso de los días de estadía los porcentajes de error son muy bajos, rondando el $1 \%$. En cambio, la situación laboral y el nivel de instrucción presentan niveles de error extremadamente altos, con alrededor del 50\% de ignorados.

Los análisis de las variaciones provinciales en la calidad del registro dan cuenta de que, en forma consistente con lo observado en el nivel de error de la variable edad de los egresos hospitalarios del total de casos del país, en todas las provincias el porcentaje de falta de información en la variable edad, es inferior al 10\%, aunque dentro de ese parámetro se advierte la heterogeneidad en la calidad de la información.

Los niveles de error del diagnóstico de egreso hospitalario presentan, en general, para todas las provincias y para la mayoría de los últimos años considerados, proporciones inferiores al $10 \%$. Situación que se mantiene tanto para el total de los egresos como para los egresos de niños de 0 a 4 años. No obstante, en cada región, se destaca alguna provincia que presenta valores disonantes en algún año.

La información referida a la cobertura de salud de los egresos hospitalarios muestra niveles de error con un comportamiento diferencial según áreas, con guarismos inferiores al $5 \%$ en las provincias de la Región de Cuyo; de alrededor del $10 \%$ en las provincias de la Región Pampeana y superiores al $20 \%$ en el resto de las regiones.

Nacimientos: Se realizó una selección de las variables disponibles en la fuente de información, sobre la base del criterio de que dichas características podrían ser potenciales insumos pertinentes para la investigación (con excepción de la variable sexo). Se analizaron los porcentajes de casos desconocidos o ignorados de cada variable como indicador de calidad de la información, para el período 1999-2012.

Las variables evaluadas fueron: provincia y departamento, referidas a la identificación de los nacimientos en el área de residencia correspondiente; sexo, peso al nacer, tipo de parto, atención del parto, local de ocurrencia, edad de la madre, nivel de instrucción de la madre y tiempo de gestación.

Se observó que la mayoría de las variables evaluadas presentan una calidad con niveles aceptables para el total del país, en el período analizado. Resulta aceptable también la calidad de las variables provincia de residencia, departamento de residencia, sexo, peso al nacer, tipo de parto, local de ocurrencia, edad de la madre, nivel de instrucción de la madre y tiempo de gestación, variables en las que se observa un notable descenso del nivel de ignorados a lo largo del período analizado, alcanzando proporciones menores al $4 \%$ en los últimos años. En cambio, la calidad de la variable 
atención del parto no es aceptable ya que se observaron fluctuaciones permanentes, durante todo el período, en el nivel de ignorados, con alzas y bajas muy pronunciadas.

La evaluación de calidad a nivel provincial concluyó que, en el caso del peso al nacer, edad y nivel de instrucción de la madre, hay disparidades y situaciones puntuales con calidad no aceptable; pero, en función del aporte que estos indicadores podrían sumar al análisis de la desnutrición en Argentina y considerando que en los últimos años la serie temporal, en general, tiende a una mejora en la calidad de la información, sería posible incorporarlos al análisis, aunque teniendo siempre presente, sobre todo para algunas provincias, el análisis de calidad. La información sobre el tiempo de gestación, en la que se observó un nivel de calidad menor, podrá ser utilizada con especial precaución, para no cometer errores de arribar a conclusiones distorsionadas. No obstante, resulta importante que, con estas consideraciones, la información, de por sí escasa, pueda ser aprovechada.

Lo que hasta aquí desarrollamos permite reflexionar sobre el potencial de la información utilizada para dar cuenta de la desnutrición en Argentina, en los primeros años del siglo XXI. El desafío fue, frente a las limitaciones de calidad evidenciadas en el análisis de los datos, extremar su potencialidad para hacer visibles los factores asociados a la desnutrición.

No cabe duda de que, para ciertas variables, los bajos niveles de calidad denegarían la posibilidad de utilizarlas. Sin embargo, hay diferentes opiniones respecto al uso de estadísticas vitales limitadas: están aquellos que dudan de la posibilidad de hacer análisis cuando la calidad es deficiente y están los que piensan que siempre es posible hacer correcciones a los datos, independientemente de su calidad. En esta controversia, y en el marco de esta propuesta, se creyó correcto considerar que ninguna información es absolutamente despreciable, aunque se debe tener conciencia de la limitación de los datos y de cuánto se puede esperar de ellos, tomándolos, en algunos casos, con suma cautela. Es en este sentido que se considera válido el desarrollo analítico de los indicadores seleccionados. Esto pone de relevancia la existencia de cierta amplitud respecto a la información disponible, que aunque debe ser mejorada en su calidad, resulta fundamental para la descripción de la patología de la desnutrición en sus formas no extremas, procurando detectar aquellas manifestaciones que aumentaron su incidencia en los primeros años de siglo XXI en Argentina.

Complementariamente al abordaje cuantitativo de la desnutrición se incluyó una vertiente cualitativa. La misma se abordó mediante la recopilación y sistematización de información referida a los Programas y Políticas Alimentarias a fin de contextualizar la preocupación del Estado por la problemática a lo largo del periodo. De igual modo, con el afán de obtener una visión actual agregada, se realizaron entrevistas a informantes calificados residentes en las provincias de Tucumán, Buenos Aires y Ciudad Autónoma de Buenos Aires. Dichas entrevistas fueron semiestructuradas y se dividieron en cinco secciones generales, y estuvieron elaboradas teniendo como horizonte de fondo la problemática de la desnutrición infantil y el perfil de los entrevistados. Se estableció la inclusión de un bloque inicial de preguntas, que caracterizan a la institución de referencia y la identidad del informante, y un bloque de preguntas sobre la población de referencia, demandas y necesidades. Se adoptó el supuesto de que los entrevistadores-investigadores conocen el perfil del entrevistado, los programas que se aplican en el área de referencia y los datos disponibles respecto de la desnutrición. El universo de posibles entrevistados estuvo conformado por funcionarios responsables del diseño, evaluación o ejecución de programas alimentarios; responsables de instituciones y referentes de la temática (hospitales, comedores y ONG, entre otros) que colaboren con sus conocimientos y evaluaciones sobre el problema en sus áreas de intervención o competencia. También se extendió a expertos de las áreas de nutrición y pediatría, para consultarles sobre su visión general de las prioridades del país, los programas y recomendaciones y sus percepciones en cuanto a los principales problemas de salud y las brechas en el conocimiento.

Ambas estrategias aportaron una visión complementaria de la desnutrición en Argentina que proporcionan elementos para direccionar estrategias de acción que busquen trabajar en su resolución en forma más acabada.

\section{Resultados}

Si se considera la expresión más trágica de la desnutrición infantil, es decir la muerte por esta causa, se presentaba en Argentina un panorama preocupante. En la Figura 1 se observa que la tendencia de la mortalidad de los menores de 5 años por desnutrición muestra en el transcurso de la década un sostenido descenso luego de la crisis de los años 2002-2003, cuando alcanzó un pico de 0,07 por mil. A partir del año 2003 se detecta un descenso sostenido hasta alcanzar en el año 2013 una tasa de 0,01 por mil. Esto implicó un descenso relativo del $80,6 \%$, lo que podría constituir ante una primera impresión un hecho altamente auspicioso. 
Figura 1. República Argentina. Tasa de mortalidad de menores de cinco años por desnutrición y otras anemias nutricionales (1999-2013).

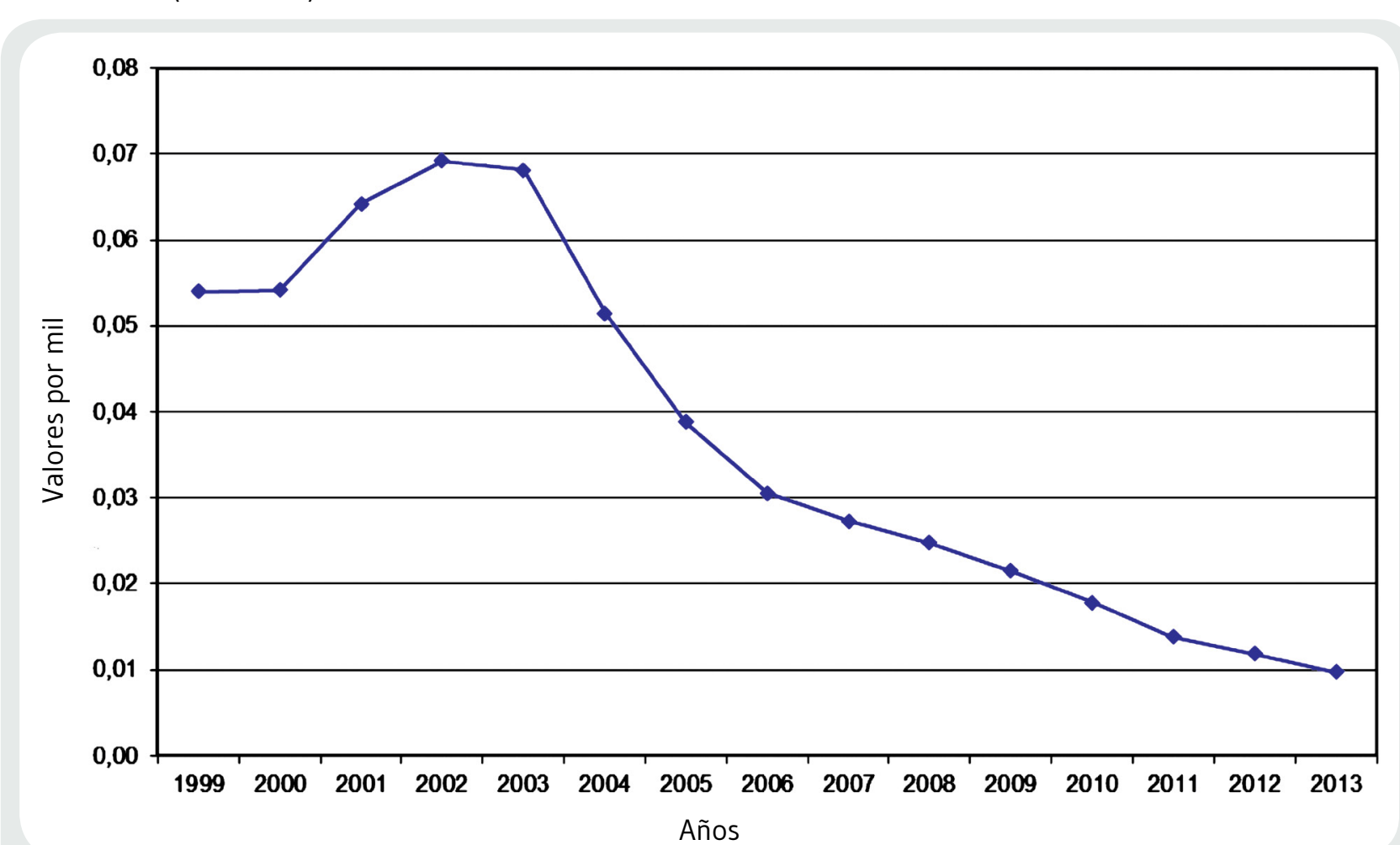

Fuente: Programa Nacional de Estadísticas de Salud, Dirección de Estadísticas e Información de Salud, Ministerio de Salud de la Nación.

En todo el país, en este periodo histórico designado por algunos autores como neodesarrollismo, ocurrieron 1951 muertes de niños menores de 5 años registradas por esta causa. Se conjetura además que hubo un universo importante de muertes subregistradas o registradas bajo otra causa, lo cual acrecentaría el panorama descripto en cuanto a la muerte infantil por desnutrición. El neodesarrollismo asume características de un orden diferente al neoliberal. En Argentina, luego de poco más de una década signada por el proceso neoliberal, en la que -existe un amplio consenso- los resultados en términos de desigualdad fueron realmente preocupantes, las ideas de esta nueva concepción acerca de la forma en que debían organizarse la sociedad y el territorio parecieron constituir un bálsamo que permitiría iniciar el camino hacia un desarrollo genuino, pero que sobre todo ayudaría primero a morigerar, para luego casi eliminar, las enormes diferencias regionales existentes en el país.

Sin embargo, esta condición promedio del país que mencionamos implica además un gran abanico de situaciones.
Encontramos provincias como Tierra del Fuego donde la muerte infantil por desnutrición fue un hecho ausente en todo el periodo, hasta provincias como Salta, donde ocurrieron 48 defunciones según estas características, y la hizo ocupar el pico histórico del periodo en el año 2002.

Estos valores llevaron a Salta a alcanzar una tasa de mortalidad por desnutrición infantil en 2002 de 0,30 por mil, constituyendo el valor más alto alcanzado en el periodo de estudio en el conjunto de provincias del Noroeste argentino. Una situación más severa se hallaba en las jurisdicciones del Nordeste argentino, donde Formosa detentaba una tasa de 0,67 por mil y Chaco de 0,36 por mil en el mismo año, mostrando en ellas una mayor severidad del problema.

Cabe aclarar además que esta misma provincia, reunió en los quince años analizados, 323 defunciones de menores de 5 años por desnutrición, un panorama epidemiológico que se asocia fuertemente a la persistencia y magnitud que alcanza la pobreza entre las provincias del Norte argentino. 
No obstante, la muerte no es la única consecuencia de la desnutrición; existen muchos niños que la padecen y la sufren, y aunque no llegan a morir, las secuelas dejadas en ellos generan daños en distintos aspectos de su interacción social. La morbilidad por esta causa adquiere una magnitud importante también en Argentina, y como se mencionó anteriormente, hay escasas fuentes que permiten aproximarnos a esta manifestación de la desnutrición infantil.

Poniendo atención en este punto, exploramos aquí una fuente poco usada en términos nutricionales para abordar esta problemática, usando así el registro nacional de Estadísticas Hospitalarias. Esta fuente, con las limitaciones que se han mencionado, permitió aproximarnos al objetivo de detectar las características y evolución de la morbilidad por desnutrición. No obstante, deseamos hacer énfasis en que esta fuente permitió una mirada sobre un aspecto de la morbilidad, es decir el egreso hospitalario, soslayando del análisis todo aquel niño con desnutrición no hospitalizado (u hospitalizado y registrado bajo otra causa).
La curva de los egresos hospitalarios por desnutrición en la niñez (Figura 2) presenta valores notoriamente más elevados que la mortalidad. En este caso el registro supera entre diecinueve y cuarenta y cinco veces el valor de la mortalidad (según el año que se considere). Dicha tendencia muestra también un descenso, aunque menos pronunciado que el caso anterior.

En esta variable la curva pasó de un valor de 1,03 por mil en el año 2000 a 0,62 por mil en 2011, lo cual representa un descenso relativo del orden del $40 \%$. Al igual que en el análisis de la mortalidad hubo una importante variedad de casos provinciales, cuyos extremos están representados nuevamente por las provincias de Salta y Tierra del Fuego, donde en términos absolutos se contabilizaron 416 egresos infantiles por desnutrición infantil en el año 2000 (en el caso salteño) y valores que oscilaron entre 1 y 3 en el caso fueguino. En ambos casos totalizan en los años analizados 2.081 y 5 egresos en la niñez por desnutrición respectivamente. Sin embargo, al analizar las tasas calculadas, los valores más elevados los hallamos en las provincias de Chaco

Figura 2. República Argentina. Tasa de egresos hospitalarios de menores de cinco años por desnutrición y otras anemias nutricionales (2000-2011).

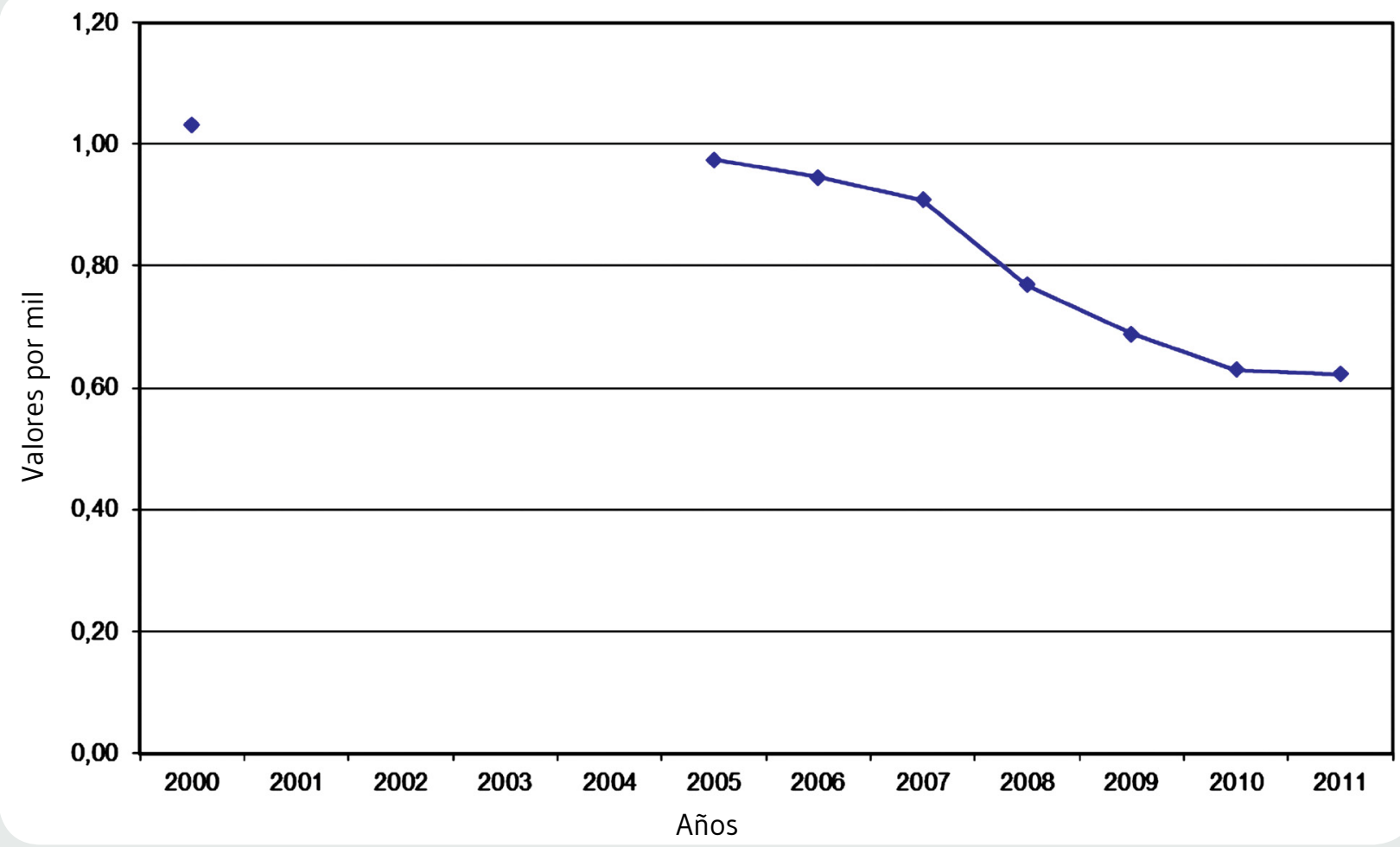

Fuente: Programa Nacional de Estadísticas de Salud, Dirección de Estadísticas e Información de Salud, Ministerio de Salud de la Nación. 
con una tasa de 3,25 por mil en el año 2000, seguida por Salta con 3,17 por mil en el mismo año.

Ambos indicadores de la desnutrición infantil -mortalidad y egresos hospitalarios- junto a otras evidencias antropométricas o bioquímicas brindan argumentos para destacar la magnitud que alcanza la desnutrición infantil, a pesar del descenso operado, en el contexto argentino en general y en el Norte del país en particular, los cuales necesitan abordajes prioritarios y políticas específicas para la generación de información y su posterior diagnóstico.

Una exploración metodológica particular en el estudio de la desnutrición infantil resultó aquella relacionada con las estadísticas de nacidos vivos según variables relacionadas al peso del niño/a, a la educación de la madre y a la duración del embarazo. En este sentido se calculó la proporción que representan los nacidos vivos con menos de $2.500 \mathrm{~g}$, provenientes de madres con educación crítica (primario incompleto) y con gestaciones a término (iguales o superiores a las 37 semanas). La proporción de los nacimientos de bajo peso presenta también un notorio descenso, con registros del orden del 4\% en 1999 a un 1,5\% en 2012 (Figura 3). Se observa un quiebre en la tendencia alrededor del año 2007, la cual vuelve a retomar luego de ese año un recorrido descendente.

Resulta evidente la importante brecha que existe entre las incidencias de cada uno de los indicadores, lo cual provee supuestos sobre la amplitud potencial del problema de la desnutrición, magnitud que resulta desconocida tanto en los ámbitos académicos como políticos actuales.

Se puede afirmar, entonces, que aun cuando pueda existir algún nivel de subregistro en las defunciones ocurridas estrictamente por desnutrición, las otras variables que también registran el fenómeno muestran la misma tendencia. De esta manera se observan dos resultados principales: una clara tendencia descendente del problema y una polarizada distribución al interior de las provincias.

Figura 3. República Argentina. Proporción de nacimientos con bajo peso según edad gestacional y baja educación de la madres (1999-2012).

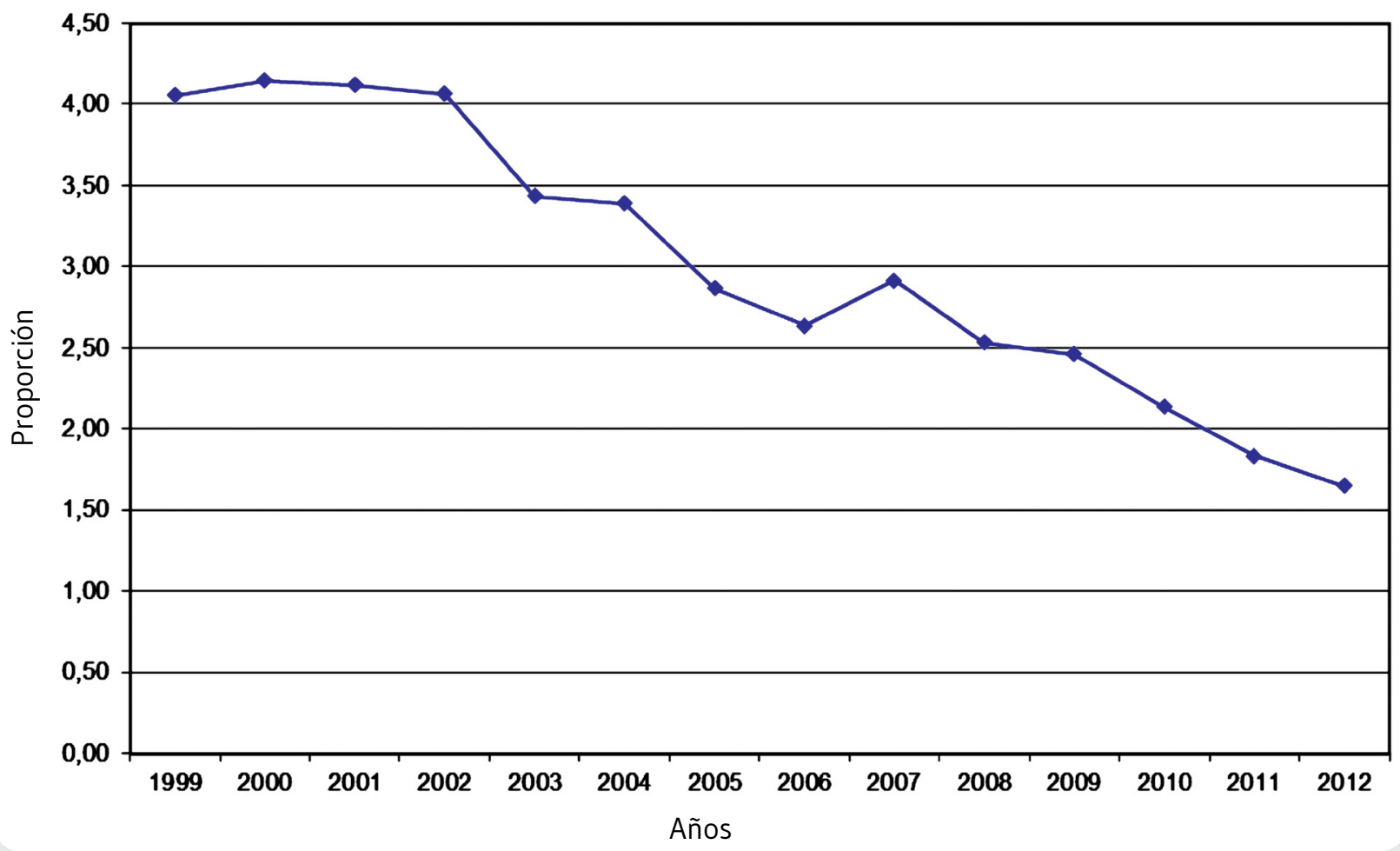

Fuente: Programa Nacional de Estadísticas de Salud, Dirección de Estadísticas e Información de Salud, Ministerio de Salud de la Nación. 


\section{La escala regional}

En relación a este último punto existe una clara diferenciación regional tanto en las magnitudes como en las tendencias que alcanza la desnutrición en la niñez según los indicadores seleccionados (Figuras 4, 5 y 6 ). En todos los casos, el NEA (Nordeste argentino) y el NOA (Noroeste argentino) son las regiones que presentan las peores condiciones, aunque los comportamientos de cada una de las manifestaciones de la desnutrición son muy disímiles.

Mientras que la mortalidad ya había sido controlada durante los noventa en buena parte del país, en el Norte constituía -ya avanzado en el siglo XXI- todavía un problema no resuelto. Hubo allí avances significativos durante la primera década del presente siglo, sobre todo luego de la crisis que atravesó el país en 2001. Hacia 2013, aun cuando el NEA presentaba una brecha importante con respecto al promedio nacional, podía observarse una tendencia a la convergencia en los niveles regionales.
La morbilidad presentaba un comportamiento mucho más estable en su tendencia (Figura 5). Asimismo, la diferenciación regional observada en la mortalidad era aún más notoria en este caso. El valor absoluto que alcanzaron los egresos por desnutrición en el periodo alcanzó a 15.684 menores de 5 años, lo cual representa el 0,69\% de los egresos hospitalarios ocurridos de niños en ese tramo de edad. Una simple relación estadística muestra que por cada muerte de un niño por desnutrición ocurren 8 egresos.

Esta vertiente del problema que buscamos caracterizar muestra un aspecto que permanece oculto en el indicador tradicional precedentemente analizado. Observamos que la región Metropolitana, sobre mediados del periodo, alcanzó los valores más elevados, descendiendo a partir de entonces para ubicarse detrás del NOA y NEA como la tercera región con mayores tasas de egreso por desnutrición del país.

Figura 4. Regiones argentinas. Tasa de mortalidad de menores de cinco años por desnutrición y otras anemias nutricionales (1999-2013).

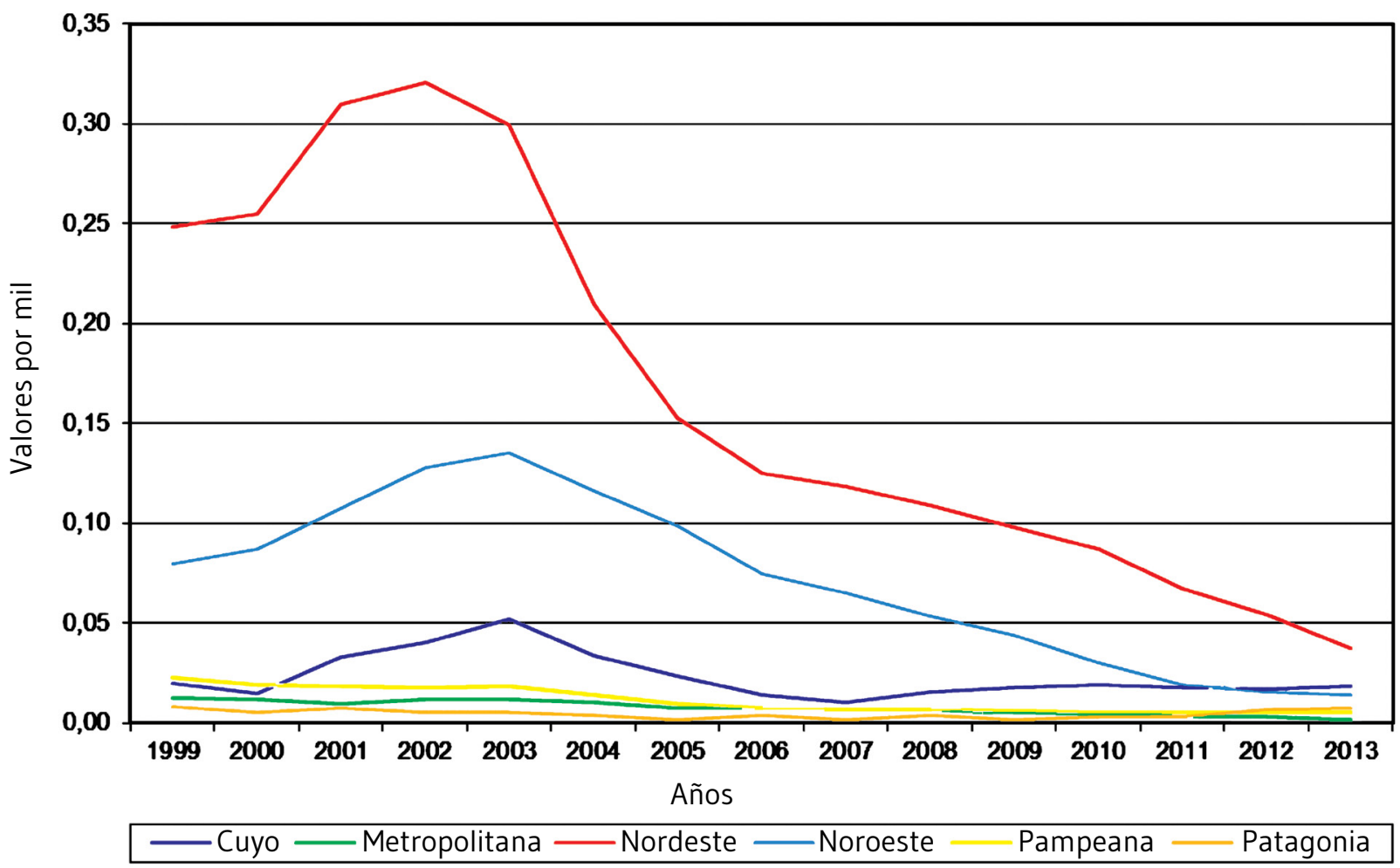

Fuente: Programa Nacional de Estadísticas de Salud, Dirección de Estadísticas e Información de Salud, Ministerio de Salud de la Nación. 
La vertiente del bajo peso al nacer ayuda a comprender mejor cuáles son las magnitudes de las diferencias regionales y, aunque también evidenció una tendencia descendente al igual que los aspectos precedentemente analizados, puede verse claramente que el NOA y el NEA registraron los valores más altos del país.

\section{El análisis cualitativo}

El análisis de los Programas y políticas alimentarias evidencia que desde fines del siglo $X X$ y fundamentalmente en las primeras décadas del XXI se impulsaron programas, tanto desde formas rudimentarias hasta sistemas más formales de ayuda o asistencia alimentaria, que en forma sucesiva pasaron del enfoque asistencialista universal al focalizado. La recopilación de la información sobre la temática puso de manifiesto que desde los años ' 80 hasta la segunda década del siglo XXI se realizaron políticas y programas con diferentes modalidades y criterios que fueron promovidos por las áreas ministeriales de Desarrollo Social y de Salud.
En el ámbito de Desarrollo Social, entre los programas iniciados antes de la década del 2000 se destacan el Programa Alimentario Nacional (1983), Bono solidario (1989) y Programa de Ayuda Solidaria de Emergencia (1990). No cabe duda que existieron numerosos programas destinados a sostener la demanda de alimentos y el financiamiento de familias vulnerables cuya principal característica era su descentralización. Asimismo, fueron financiados por organismos internacionales como el Banco Mundial y Programa de las Naciones Unidas para el Desarrollo (PNUD). La principal particularidad de todos estos consistió en una asistencia técnica y financiera directa, en general a través de la entrega de cajas o bolsones de alimentos.

Un punto de inflexión ocurrió a partir del año 2003, cuando -a partir de la estrepitosa crisis de hambre y desnutrición del año 2001- se implementa el PNSA (Plan Nacional de Seguridad Alimentaria) como un programa de alcance universal, no focalizado (aunque la población a la que atiende es aquella de bajos recursos y zonas

Figura 5. Regiones argentinas. Tasa de egresos hospitalarios de menores de cinco años por desnutrición y otras anemias nutricionales (2000-2011).

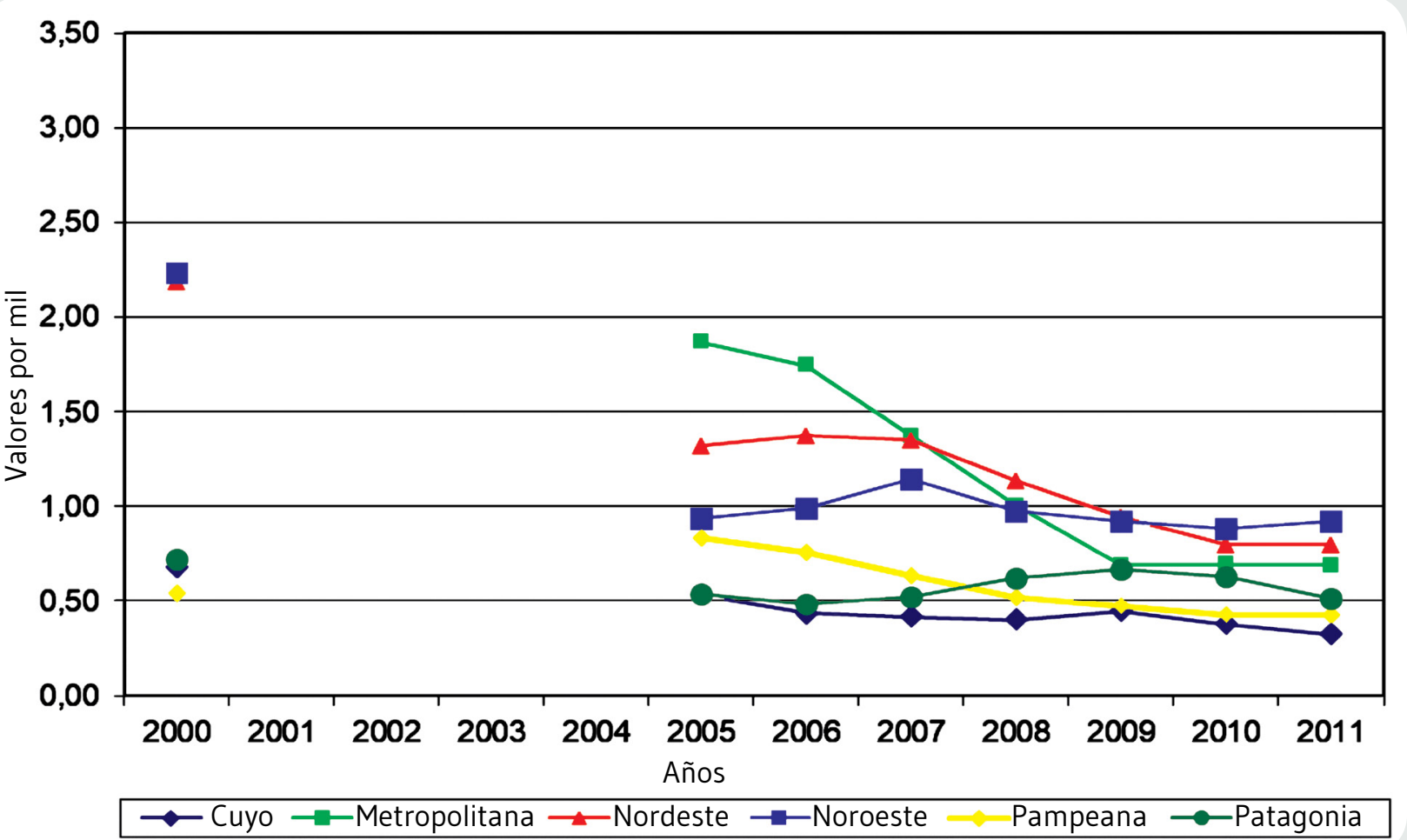

Fuente: Programa Nacional de Estadísticas de Salud, Dirección de Estadísticas e Información de Salud, Ministerio de Salud de la Nación. 
Figura 6. Regiones argentinas. Proporción de bajo peso al nacer según edad gestacional y baja educación de la madre (1999-2012).

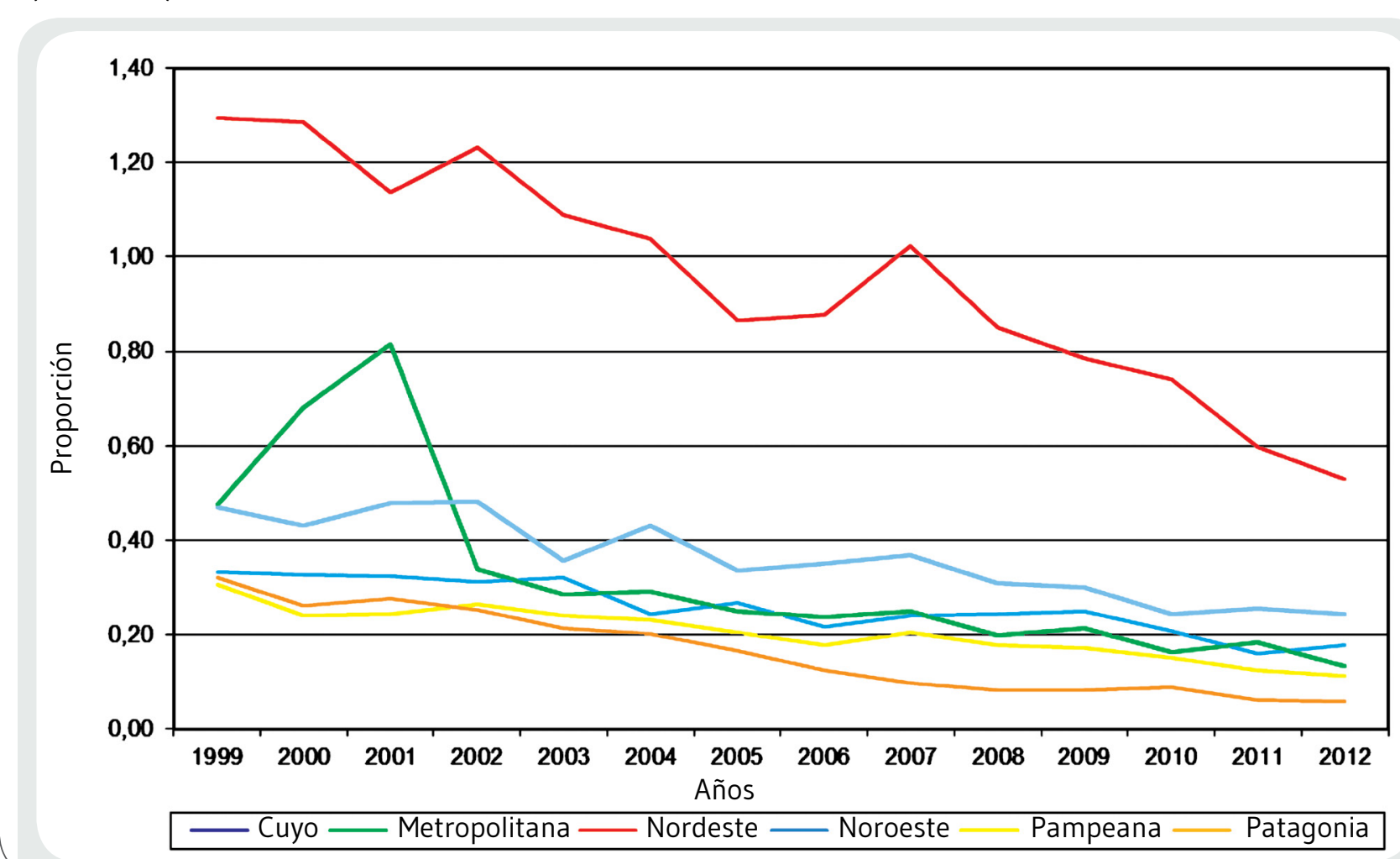

Fuente: Programa Nacional de Estadísticas de Salud, Dirección de Estadísticas e Información de Salud, Ministerio de Salud de la Nación.

desfavorecidas), el cual operaba a través de los comedores comunitarios, el desarrollo de huertas, la capacitación y la promoción del entorno saludable. De estos componentes, el mayor presupuesto estuvo destinado a asistencia alimentaria.

Paralelamente, la implementación de tarjetas magnéticas asociadas a cuentas donde el Estado transfiere dinero para la compra de alimentos estableció un corte respecto de anteriores programas de ayuda alimentaria. Se promovió la compra de alimentos frescos (en contrapartida con las cajas de anteriores programas donde el componente mayoritario eran alimentos secos y no perecederos). Esto se acompaña de acciones de capacitación y promoción no sólo a los agentes intervinientes sino también a las familias; todo esto como parte del proceso de cambio cultural respecto de la importancia de la alimentación. EI PNSA significó un cambio de paradigma respecto a la década precedente al ser más extensivo e integrar varios componentes (educativos, alimentarios, sociales, comunitarios, etc.).
Los programas promovidos del área de Salud han seguido el mismo camino: aquellos que se iniciaron en décadas previas continuaron y ampliaron su cobertura (como los Materno Infantiles) pero se tendió a universalizar la cobertura de salud en varios sentidos: planes como Remediar para la obtención de medicamentos, los Centros de Atención Primaria que tienden a descentralizar la atención básica, la mayor cobertura de salud a través de programas como el Sumar y los cambios en la política sanitaria de prevención con las modificaciones en el calendario de vacunación obligatoria han sido medidas tendientes a la ampliación de la base de beneficios brindados por el Estado en esta materia.

A lo anterior debe sumarse los programas de orden general como la Asignación Universal por Hijo (AUH) o Asignación Universal por Embarazo (AUE) por el efecto que ambos han tenido sobre los ingresos corrientes de las familias.

Como resultado de estas revisiones, se observó una creciente extensión de los alcances de estos programas en 
términos de extensión a la población y dimensiones a cubrir, tendientes a integrar la alimentación, la salud y la educación. Al tiempo que se vislumbró la carencia de estudios pormenorizados, tanto de diagnóstico de la situación al inicio de su implementación como de evaluaciones sistemáticas e institucionalizadas de resultados o de impacto, que permitan medir el verdadero alcance de estas políticas. La insuficiencia de esta información no permite realizar evaluaciones de proceso que posibiliten vislumbrar si tales planes han sido efectivos a la hora de combatir el flagelo de la desnutrición.

Paralelamente, las entrevistas realizadas han corroborado lo observado en el análisis cuantitativo, en relación con el descenso de la desnutrición severa en los últimos años y una mejora en algunos indicadores. Se realizaron en total ocho entrevistas a informantes calificados que trabajan en forma directa con la implementación de programas alimentarios, o bien en la atención en áreas de alta probabilidad de detección de situaciones de desnutrición infantil. Sin embargo, se advierte a través de las palabras de estos informantes, que hay aún mucho por modificar para que el problema tenga vías de solución.

Entre las causas más frecuentes se destacan las particularidades sociales en que vive la población expuesta al riesgo: la pobreza y la vulnerabilidad del medio en el que desenvuelven sus vidas los niños y niñas, tanto en lo referente al hábitat como al entorno social de esas familias. La presencia de viviendas precarias, barrios sin acceso a servicios básicos, falta de educación alimentaria, violencia familiar y consumo de drogas aparecen entre los condicionantes más referidos.

Si bien se reconoce la existencia de un mayor acceso a alimentos por la mejora en los ingresos -tanto por trabajo como por programas universales como la AUH- persiste una baja calidad de alimentación en términos de nutrientes que son vitales para el crecimiento y desarrollo adecuado. La desnutrición oculta (Ilamada así a la carencia de hierro) se relacionaría específicamente con esta problemática.

La dificultad que mencionan todos los entrevistados y que parece ser también soslayado por las políticas públicas actuales es el problema del sobrepeso y la obesidad, ambos como consecuencia de una mala administración en los recursos alimentarios que se brindan, así como una ausencia de análisis culturales y de una política deliberada de cambio educativo que permita una transformación radical en el modo en el que se maneja la compra, acceso, cocción y almacenamiento de las comidas, así como las características de los entornos saludables y la educación física y para la salud.
Otra cuestión es la organización interna de la gestión orientada a la resolución del problema en los centros de salud y atención comunitaria, donde se destacan como exitosas aquellas experiencias en que los entrevistados han diseñado o participado en los equipos interdisciplinarios. Asimismo, se menciona la necesidad de crear instancias intra-gubernamentales que pueden producir sinergia positiva para la ejecución de los programas.

Como propuestas más importantes se señalan la necesidad de contar con diagnósticos precisos y previos a la implementación de un programa, evaluaciones de proceso para detectar el cumplimiento de los objetivos previstos en los programas, así como cálculos de impacto y cambio sobre la población. La informatización de los registros, la accesibilidad de la información, la comunicación de las distintas esferas involucradas en la ejecución de un programa y la necesidad de contar con una mirada integral son las otras mejoras que se demandan desde los actores que trabajan diariamente con esta problemática.

\section{Discusión}

La desnutrición infantil conforma el principal núcleo duro de privación y exclusión que debería ser prioritario en las políticas públicas. Existen muchas barreras para su conocimiento y análisis, que dependen a su vez de las escalas que se utilizan y las fuentes que se emplean. Si bien en algunas investigaciones previas se ha procurado detectar su magnitud ${ }^{12-15}$, asumimos aquí la propuesta de indagar sobre algunas manifestaciones actuales del problema a partir del abordaje de fuentes no convencionales.

Luego de los análisis precedentes, queda claro que cada una de las vertientes que se analizan resulta insuficiente para caracterizar el problema de la desnutrición en la niñez, pero el análisis conjunto permite obtener una caracterización y una comprensión del fenómeno mucho más clara. En este sentido, esta investigación pretendió sentar las bases para una nueva forma de enfocar el problema de la desnutrición, la cual no se agota en los aspectos aquí estudiados, pero que abre un fértil campo para estudios posteriores sobre la materia.

De una manera casi concluyente puede verse, luego de analizar el problema y de observar un sostenido descenso, que lejos de ser una cuestión del pasado, la desnutrición en la niñez constituye uno de los problemas de mayor relevancia en la actualidad, el cual, junto a la emergencia de los problemas del exceso de peso, la obesidad y las enfermedades 
crónicas no transmisibles, deberían constituir los principales núcleos de interés de la agenda pública.

Las evidencias halladas muestran a su vez una marcada desigualdad en su distribución espacial, detectando casos en provincias del Norte donde constituye un grave problema de salud pública, influyendo tanto en las altas tasas de morbilidad y mortalidad infantil como en la prevalencia de ciertas enfermedades crónicas difíciles de cuantificar ${ }^{16,17}$, donde la existencia de un factor común como la pobreza parece ser el principal determinante de esta situación ${ }^{18}$. Asimismo, el costo económico y social de la enfermedad es muy alto también (gastos hospitalarios, productividad, gastos en educación, menor capacidad laboral, etc.), y el daño cerebral que genera, también complejo de cuantificar, aunque con profundas connotaciones éticas y morales ${ }^{19,20}$.

En este trabajo, que profundizó el análisis crítico sobre las fuentes de información susceptibles de ser abordadas para estudiar este problema detectando limitaciones (subregistro, causas ignoradas o mal registradas, ausencia de establecimientos informantes, entre otros) destacamos la fortaleza que presenta la complementariedad de enfoques cuantitativos y cualitativos. Ambos detectan aspectos parciales de un problema multifacético como la desnutrición infantil, pero en un análisis complementario permiten una visión más integral. El estudio de la desnutrición infantil en sus vertientes cuantitativas (mortalidad, morbilidad y bajo peso) como cualitativa (análisis de políticas y programas como entrevistas a informantes clave) renuevan el compromiso de seguir estudiando el problema holísticamente con toda la información disponible para conocer en forma más certera la situación y propender a gestionar políticas eficaces. Este enfoque complementario aporta una visión general de las diferentes aristas del fenómeno y los posibles caminos para su abordaje. Esto sumado a la perspectiva interdisciplinaria para el abordaje del problema, fortalece la organización y la documentación efectiva de los instrumentos que permitan realizar políticas de detección, prevención y seguimiento.

\section{Conclusiones}

Los problemas nutricionales en la niñez argentina se encuentran actualmente atravesados por resabios del pasado -entre ellos la desnutrición-y la emergencia de nuevos problemas, entre los que se destacan el sobrepeso y la obesidad. Los resultados hallados constituyen un capital relevante de insumos para promover acciones concretas en materia de cuidado y protección infantil, diferenciadas según la magnitud, tendencias y localización espacial de determinados problemas nutricionales. En efecto, las provincias del Norte deberían constituir una prioridad para las políticas públicas que busquen atenuar o erradicar los actuales niveles de desnutrición en la niñez.

\section{REGISTRO DEL PROTOCOLO DE ESTUDIO}

Esta investigación ha sido registrada en el Registro Nacional de Investigaciones en Salud de Argentina (RENIS) bajo el código IS000917.

\section{FINANCIACIÓN}

Comisión Nacional Salud Investiga (Ministerio de Salud de la Nación).

\section{conflicto de intereses}

Los autores expresan que no existen conflictos de interés al redactar el manuscrito.

\section{REFERENCIAS}

(1) Mercer R. Salud y pobreza en la Argentina Dime cómo ha sido tu cuna y te diré cómo serás. Voces en el Fénix. 2013; 22.

(2) Organización de las Naciones Unidas para la alimentación y la agricultura. Panorama de la inseguridad alimentaria en América Latina y el Caribe. FAO: Roma; 2015. Disponible en http://www.fao.org/3/a-i4636s.pdf

(3) Ortiz-Andrellucchi A, Peña Quintana L, Albino Beñacar A, Mönckeberg Barros F, Serra-Majem L. Desnutrición infantil, salud y pobreza: intervención desde un programa integral. Nutr Hosp. 2006; 21(4): 533-41.

(4) Longhi F, Gomez A, Zapata M, Olmos F, Paolasso P, Ramos Margarido S. La desnutrición en la niñez argentina en los primeros años del siglo XXI: un abordaje cuantitativo. Salud colectiva. 2018; 14: 33-50.

(5) Organización Mundial de la Salud. Informe de la Comisión para acabar con la obesidad infantil. Washington: OMS. 2016 Disponible en https://apps.who.int/gb/ebwha/pdf_files/ WHA69/A69_8-sp.pdf 
(6) UNICEF Desnutrición infantil en América Latina y el Caribe. Desafíos. 2006; 2. Disponible en http://www.unicef.org/lac/ Desafiosnutricion(13).pdf.

(7) Organización de las Naciones Unidas para la alimentación y la agricultura. Perfiles nutricionales por países: Argentina. Roma: FAO; 2001. Disponible en http://www.fao.org/tempref/AG/ agn/nutrition/ncp/arg.pdf

(8) Fondo de las Naciones Unidas para la Infancia. Censo de talla de escolares de primer grado. 1991. Informe de los resultados. Buenos Aires: UNICEF, 1995.

(9) Britos S. Crisis 2001-2002: pobreza, precios y alimentos. Buenos Aires: Centro de Estudios sobre Nutrición Infantil; 2003.

(10) Calvo E, Aguirre P. Crisis de la seguridad alimentaria en la Argentina y estado nutricional en una población vulnerable. Arch Argent Pediatr. 2005; 103: 71-90.

(11) Von Elm E, Altman DG, Egger M, Pocock SJ, Gøtzsche PC, Vandenbroucke JP. Declaración de la Iniciativa STROBE (Strengthening the Reporting of Observational studies in Epidemiology): directrices para la comunicación de estudios observacionales. Gaceta Sanitaria. 2008; 22(2): 144-50.

(12) Oyhenart $E$, et al. Estado nutricional infanto juvenil en seis provincias de argentina: variación regional. Revista Argentina de Antropología Biológica. 2008; 10.

(13) Longhi F. Magnitudes y tendencias de la desnutrición en la niñez argentina durante la primera década del siglo XXI. Población y Salud en Mesoamérica. 2015; 13.
(14) Durán P, Mangialavori G, Biglieri A, Kogan L, Abeyá Gilardon E. Estudio descriptivo de la situación nutricional en niños de 6-72 meses de la República Argentina: resultados de la Encuesta Nacional de Nutrición y Salud (ENNyS). Arch Argent Pediatr. 2009; 107(5): 397-404.

(15) Bolzán A, Mercer R. Seguridad alimentaria y retardo crónico del crecimiento en niños pobres del norte argentino. Arch Argent Pediatr. 2009; 107(3): 221-8.

(16) Bolsi A, Longhi F, Paolasso P. Pobreza y mortalidad infantil en el norte grande argentino. Un aporte para la formulación de políticas públicas. Cuadernos geográficos. 2009; 45: 231-61.

(17) Buchbinder M. Mortalidad infantil y desigualdad socioeconómica en la Argentina: tendencia temporal. Arch Argent Pediatr. 2008; 106(3): 212-28.

(18) Bolsi A, Paolasso P. Geografía de la pobreza en el Norte Grande Argentino. San Miguel de Tucumán: PNUD - CONICET-UNT; 2009.

(19) O'Donnell A, Porto A. Las carencias alimentarias en el país. Su impacto sobre el desarrollo infantil. Pobreza y desarrollo infantil. Una contribución multidisciplinaria. Buenos Aires: Paidós. 2007; 141-59.

(20) Kogan L, Gilardón EA, Biglieri A. Anemia: La desnutrición oculta. Resultados de la Encuesta Nacional de Nutrición y Salud-ENNyS. Ministerio de Salud de la Nación, Argentina. 2008. Disponible en http://www.sap.org.ar/docs/ profesionales/anemia-la-desnutricion-oculta.pdf 